\title{
Dynamic changes of wetland resources based on MODIS and Landsat image data fusion
}

\author{
Fawang Ma, Qiubing Wang ${ }^{*}$ and Maoxin Zhang
}

\begin{abstract}
Given the seasonal dynamics of wetland ecosystem, limited available data, and technological method of wetland investigation, wetland evaluation cannot be accurately accessed. Although the remote sensing technology has been widely employed on wetland investigation and identification, changeable weather conditions especially cloud interference are the main barrier to acquire clear remote sensing image for wetland identification and information extraction. The combination and precision evaluation of remote sensing data with high temporal-spatial resolution ratio from moderate-resolution imaging spectroradiometer (MODIS) and Landsat were conducted using the Spatial and Temporal Adaptive Reflectance Fusion Model (STARFM): a comprehensively temporal-spatial reflectance model was built; the high-resolution image in the time series and Modified Normalized Difference Water Index were obtained. The main data obstacles in wetland resources monitoring were invalid. The typical wetland areas in Liaoning province of China were evaluated using combination algorithm and Landsat (Thematic Mapper) images. The results show that the MODIS and Landsat data can be combined well with high correlations in different wave ranges. The maximum Normalized Difference Water Index (NDWI) is 0.9678 , followed by green wave (0.9630), near-infrared wave (0.9345), and blue wave (0.9018).The wetland seasonal change of Panjin was examined using the data combination method. Normalized Difference Vegetation Index (NDVI), Normalized Difference Water Index (NDWI), and vegetation coverage index were extracted from combined images of Panjin from June 2016 to August 2016 and analyzed. Results showed that the NDVI was high in partial water area during the studied period indicating high chlorophyll contents.
\end{abstract}

Keywords: Wetlands, Dynamic changes, MODIS, Landsat, Data fusion

\section{Introduction}

Wetlands play irreplaceable roles in climate regulation, water purification, biodiversity conservation, and natural ecology balancing [1]. Wetlands provide habitats for wild animals and plants, and guarantee climate modulation, pollutant deterioration and decomposition, flood detention, water conservation, soil erosion control, oxygen release from solid carbon, and ecological nutrient cycling [2]. Wetlands offer humans abundant animal and plant resources and necessary water sources, and provide turf and other special sources [3]. However, due to natural and artificial interferences, numerous wetlands have been converted to farm lands or urban lands, and such fundamental variation of wetland properties has roused wide attention from researchers [4].

\footnotetext{
* Correspondence: wangqbsy@yahoo.com

Shenyang Agricultural University, Shenyang 110866, Liaoning Province, China
}

Remote sensing (RS) has been extensively used to investigate and identify wetland resources. RS brings about huge technical support to relevant research owing to its time/cost saving, provision of abundant information, multi-platform operation, and multi-temporal advantage [5]. Though RS largely facilitates wetland research, the changeable meteorological conditions make cloud interference one of the major barriers against the acquisition of clear RS images, identification of wetlands, and information extraction. Especially, the cloudy conditions at the growing season (June to September) certainly complicate the extraction of wetland-related information (in particular, marsh wetlands). Though there are some cloud processing techniques, they are limited by image distortion and information loss [6]. In the field of wetland monitoring, the most used and mature RS source is Landsat Thematic Mapper (TM). Despite its 
spatial resolution up to $30 \mathrm{~m}$, its visiting period is only 16 days, and the occurrence of cloudy or raining weather would largely reduce the usability of TM data [7]. Especially for wetlands, the variational climates and cloudy or rainy weathers occur more frequently at the high-flow period, when the RS data become less available. Nevertheless, this limitation can be supplemented by the National Aeronautics and Space Administration (NASA) Terra moderate-resolution imaging spectroradiometer (MODIS) sensor, which provides data anywhere nearly every day and is complementary with Landsat Thematic Mapper (TM). Thus, MODIS data largely improves the possibility of acquiring rain- and cloud-free RS images on the polar-orbiting platform [8]. However, the spatial resolutions of MODIS are very low (only 250-1000 m), which are unsatisfactory for wetland resource monitoring at the medium or high scale [9].

If the high-spatial-resolution data of Landsat TM and the high-temporal-resolution data of MODIS can be effectively fused, we could obtain a time series of Landsat TM images with high space-time resolutions simultaneously, which would largely improve the reasonableness and precision in regional dynamic monitoring of wetland resources. In recent years, the space-time fusing RS techniques and different RS sources have been developed rapidly. Researchers have constantly studied effective RS data fusing techniques, aiming to acquire high-space-time resolution RS data. Gao et al. [10] studied the space-time fusion of Landsat and MODIS data and thereby established a Spatial and Temporal Adaptive Reflectance Fusion Model (STARFM), effectiveness of which was validated by relevant tests. Later, the defects and limitations of STARFM were modified. Zhang et al. [11] established an Enhanced Spatial and Temporal Adaptive Reflectance Fusion Model (STARFM). Wu et al. [12] used a hybrid pixel decomposition method, Spatial and Temporal Data Fusion Model (STDFM), to efficiently fuse MODIS and TM images. Based on STARFM, some scholars built an Enhanced Spatial and Temporal Data Fusion Model (ESTDFM), which was contributive to improving fusion algorithms [13]. Xie et al. [14] used the Combination of Downscaling Mixed Pixel Algorithm and Spatial and Temporal Adaptive Reflectance Fusion Model (CDSTRFMA) into RS data fusion. It was found CDSTARFM outperformed both STARFM and the downscaling mixed pixel algorithm with a $2 \%$ higher precision. Walker et al. [15] used STARFM to fuse Landsat and MODIS data and effectively characterized the typical phenology of forest vegetation in arid regions. Jia et al. [16] studied the classification mapping of land covers in Beijing, or namely fused Landsat Normalized Difference Vegetation Index (NDVI) time series using STARFM.

In all, STARFM has been preliminarily applied and experimentally improved since its emergence. However, the concrete applications of STARFM still require the algorithmic improvement according to the characteristics of the study areas. In this study, the study area was located in the east of Liaoning province, China. We utilized the complementary advantages between MODIS and Landsat images in time and spatial resolutions, and adopted a progressive band algorithm. Through the comparison of fusion precision, the algorithm feasible for data fusion in the study area was screened out and its precision was evaluated. The seasonal changes of NDVI, Normalized Difference Water Index (NDWI), and vegetation richness index in wetlands of Panjin city were studied, which theoretically underlie the research on dynamic changes of wetland resources in scientific rational zones.

\section{Method}

When RS data were fused using STARFM and geometric calibration and atmospheric correction with low errors were conducted, the MODIS images at period $t_{k}$ in the study area were obtained from the weighted averages of Landsat images at near time points. Meanwhile, the differences between two sources of RS in spectra, temporal resolutions and illumination conditions, upon imaging would result in certain deviation $\varepsilon_{k}$ in the reflectivity. In this regard, we supposed the ground coverage type at $\left(x_{i}\right.$, $y_{i}$ ) and the different systematic errors were unchanged with time, or namely $\varepsilon_{\mathrm{k}}$ was constant. The reflectivity on the Landsat images at time $t_{n}$ can be determined from the weighted average of MODIS data at the same time and the Landsat and MODIS images at time $t_{k}$, based on STARFM as follows:

$$
L\left(x_{i}, y_{i}, t_{n}\right)=M\left(x_{i}, y_{j}, t_{n}\right)+L\left(x_{i}, y_{j}, t_{k}\right)-M\left(x_{i}, y_{j}, t_{k}\right)
$$

where $L$ and $M$ are the pixels of Landsat and MODIS images, respectively.

To improve the prediction accuracy, we selected adjacent pixels with the same spectral properties as auxiliary information. Meanwhile, the adjacent window centering at the predicted pixel was selected and the inside pixels were convolutionally treated using a weighed function $W$, which resulted in the predicted value of the central pixel. Finally, the whole image was operated using a slide convolutional window, forming the predicted image as follows:

$$
\begin{aligned}
L\left(x_{w / 2}, y_{w / 2}, t_{n}\right) & =\sum_{i=1}^{w} \sum_{j=1}^{w} W_{i j k}\left(M\left(x_{i}, y_{j} t_{n}\right)\right. \\
& \left.+L\left(x_{i}, y_{j}, t_{k}\right)-M\left(x_{i}, y_{j}, t_{k}\right)\right)
\end{aligned}
$$

where $w$ is the width of the slide window; $L\left(x_{w} / 2, y_{w} / 2\right.$, $\left.t_{n}\right)$ is the value of the central pixel at time $t_{n}$. The 
weighting function plays a critical role, as it decides the contributions of the pixels inside the sliding window to the fused data. In practice, the weighting function of STARFM can be determined according to three indices of spectral distance, time distance, and space distance.

The spectral distance can be computed as the difference between pixel values of Landsat and MODIS images at time $t_{k}$ and specific position as follows:

$$
S_{i j}=\left|L\left(x_{i}, y_{j}, t_{k}\right)-M\left(x_{i}, y_{j}, t_{k}\right)\right|
$$

The time distance can be calculated as the difference between pixel values of MODIS at time $t_{n}$ and MODIS at time $t_{k}$ at a specific position as follows:

$$
T_{i j}=\left|M\left(x_{i}, y_{j}, t_{n}\right)-M\left(x_{i}, y_{j}, t_{k}\right)\right|
$$

The space distance can be computed as the Euclidian distance between the central pixel and the adjacent pixel in the window as follows:

$$
d_{i j}=\sqrt{\left(x_{w / 2}-x_{1}\right)^{2}+\left(y_{w / 2}-y_{j}\right)^{2}}
$$

\section{Discussion and experimental results}

\subsection{Data fusion experiments}

The MODIS data used here were mainly MODO9A1 synthesized from the 8 days of ground reflectivity and the Landsat5 TM data. During the merging process, the number of ground types in the study area should be as many as possible. In this study, the selected ground covers mainly included vegetation, water, construction land, and wetland. Since the progressive band algorithm used here was very slow, we selected the typical region in east Liaoning in the fusing algorithm and adopted the TM images in this region for precision evaluation.

The fusion between MODIS and Landsat data was mainly based on STARFM or modified STARFM, with the following basic principles. Firstly, the two phases of low-resolution MODIS images at time $t_{0}$ and $t_{k}$ were downscaled, and then, the medium-resolution Landsat image at time $t_{k}$ was used to forecast the medium-resolution image at time $t_{0}$. The key for data fusion by STARFM is how to determine weight space information and thereby this weight algorithm can automatically adapt to different regions [17]. By screening out the pixel with a similar spectrum as the central pixel, we could weight-compute the central pixel at the prediction phase by using the spectral differences, time difference of the similar pixel, and its relative distance from the central pixel. The improved STARFM basically inherits STARFM, and the data fusion process is still to calculate the central pixel at the prediction period by weighting the similarity pixels in the window. The similarity pixels of the central pixel in the window can be decided according to the threshold $\theta b$ at different bands of Landsat at time $t_{k}$. Specifically, the pixels in the window having the absolute difference from the central pixel smaller than the threshold $\theta b$ at each wave band are selected as the similarity pixels for image fusion. The fused results are listed in Fig. 1.

Clearly, the predicted images based on STARFM were significantly improved in terms of spatial resolution from the perspective of vision, and the visual effect was more ideal. To better evaluate the high-space-time resolution MODIS images predicted by STARFM, we quantitatively assessed its precision.

\subsection{Evaluation of fusion precision}

Since the TM data were stopped by 2014 , we only evaluated the data in 2016. The TM images (merged data) and the STARFM-predicted fused data in 16 August 2016 were regressed at blue, green, red, and near-infrared bands in terms of reflectivity (Fig. 2).

Clearly, as for blue, green, red, and near-infrared bands, the high-space-time resolution data predicted by STARFM are all linearly correlated with the real data. To more correctly describe the validity of the space-time fusing algorithm, we extracted NDVI and NDWI for fitting analysis (Fig. 3).

Clearly, the NDWI and NDVI from the fusing algorithm are both highly correlated with the real data. The predicted data and TM data were superposed on ENVI, and the correlations at all bands were determined using a statistical tool. Finally, the correlations between merged MODIS data and TM data at each wave band and the same date were determined. High correlations were found at all wave bands. In particular, the correlations of NDWI rank as red band $(0.9678)>$ green band $(0.9630)>$ near-infrared $(0.9345)>$ blue band $(0.9018)$, which are all very high.

Clearly, the fusing algorithm effectively solves the defects of MODIS and Landsat images and makes full use of their advantages. This algorithm can be very effective in RS-based land resource inspection and offers a scientific method for studying the severe seasonal changes of wetland resources.

\section{Application of data fusion method in wetland change}

\subsection{Study area}

Panjin (E $\left.121^{\circ} 25^{\prime}-122^{\circ} 31^{\prime \prime}, \mathrm{N} 40^{\circ} 39^{\prime}-41^{\circ} 27^{\prime \prime}\right)$ is located at southwest of Liaoning province in China, the north bank of Bohai Sea, and the center of Liao River delta and has a coastline of $118 \mathrm{~km}$ (Fig. 4). Panjin enjoys an average altitude of $4 \mathrm{~m}$ and gentle terrains, and the highest and lowest elevations are 18.2 and $0.3 \mathrm{~m}$, respectively. It has in total 21 big or small rivers. The Shuangtai 


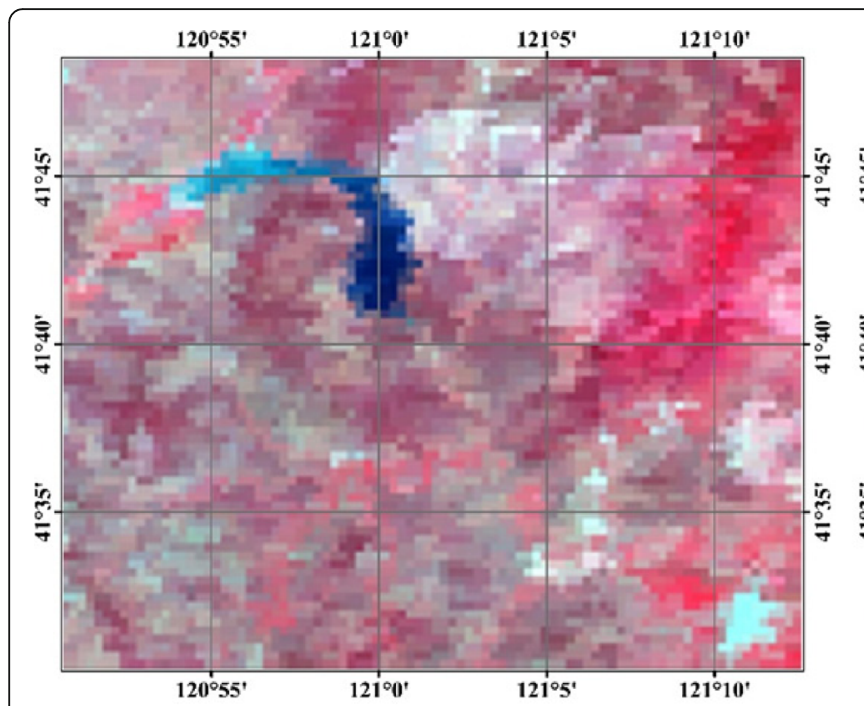

a

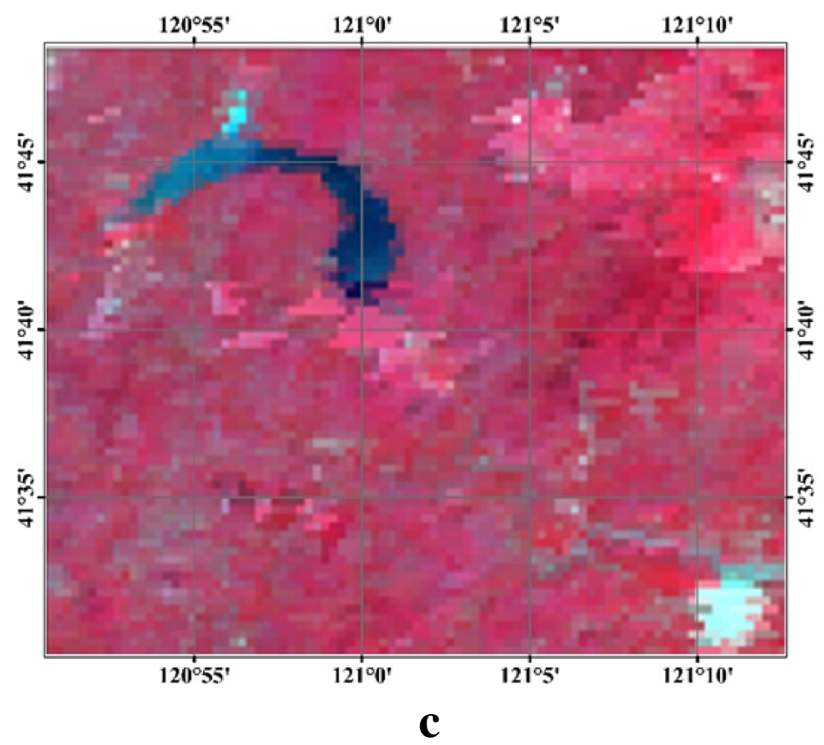

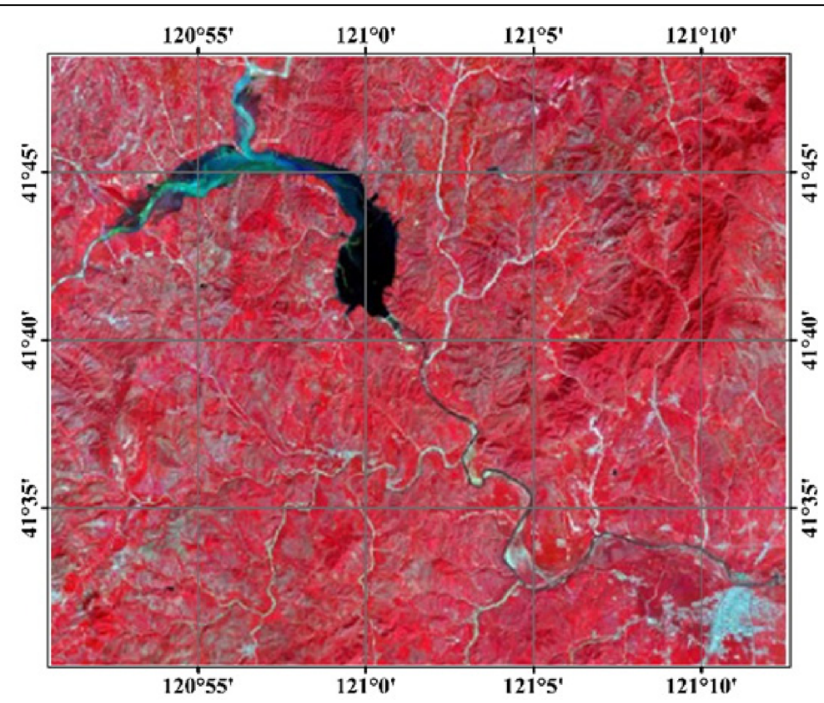

b

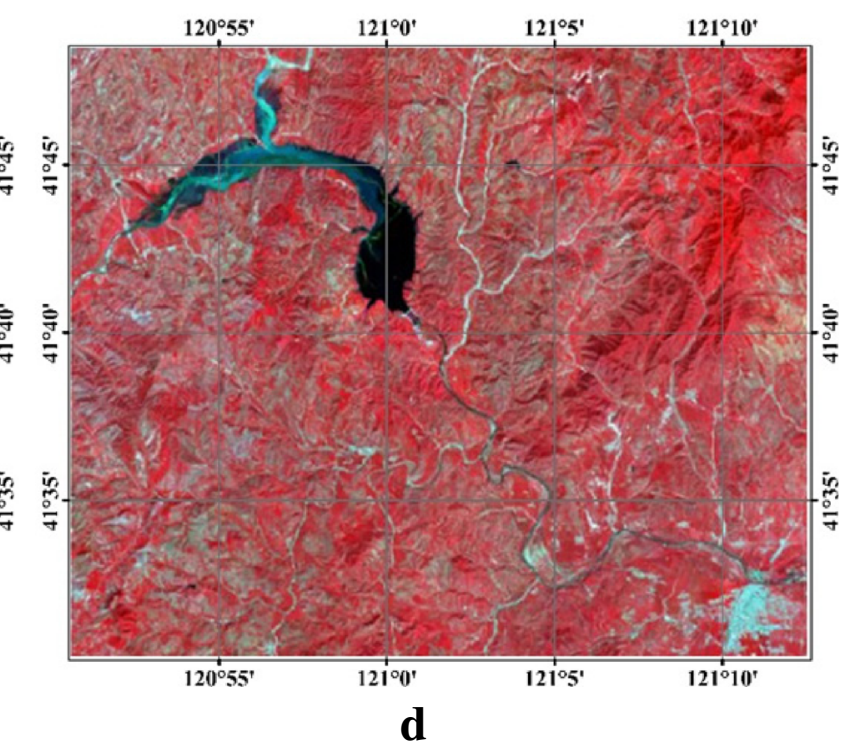

Fig. 1 The MODIS data fusion and the fusion data after false color composite in sample region. a Original MODIS image in 2016. b Fused false color synthesis in 2016. c Original MODIS image in 2014. d Fused false color synthesis in 2014

river mouth wetland nature reserve (E $121^{\circ} 28^{\prime} 10^{\prime \prime}-122^{\circ}$ $\left.00^{\prime} 24^{\prime \prime}, \mathrm{N} 40^{\circ} 45^{\prime} 00^{\prime \prime}-41^{\circ} 08^{\prime} 50^{\prime \prime}\right)$ has a total area of $4084 \mathrm{~km}^{2}$ and has been formed after the Shuangtaizi River flows to seas.

\subsection{Data fusion of the study area}

Through extraction and superposition, we analyzed the changes of NDVI and NDWI between two time phases in Panjin, as well as the changes of vegetation coverage index. Vegetation coverage measures the proportion of vertical projected areas of leaves, stems, and branches to the total area of the study site. Vegetation coverage well reflects the seasonal changes of vegetation growth, ecological environment, water/soil quality, and water conditions in wetlands and is a favorable indicator of seasonal changes in wetlands $[18,19]$.

The modified STARFM was used to fuse and predict the RS images at two phases of 2016 in Panjin (Fig. 5).

Clearly, based on the high-space-time resolution MODIS data determined from the space-time fusing algorithm, the RS data were effectively improved at both spatial and temporal scales. The image quality improvement from the perspective of data source can theoretically and efficiently improve the precision of information extraction.

\subsection{Changes of NDVI and NDWI}

NDVI and NDWI were extracted from the merged images of Panjin in June and August 2016, and the changes 


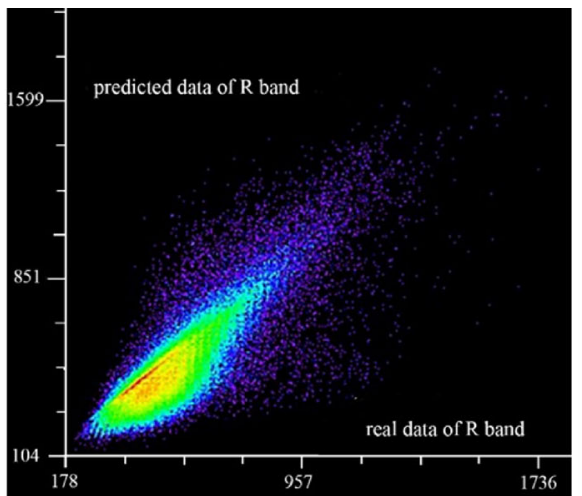

$\mathbf{a}$

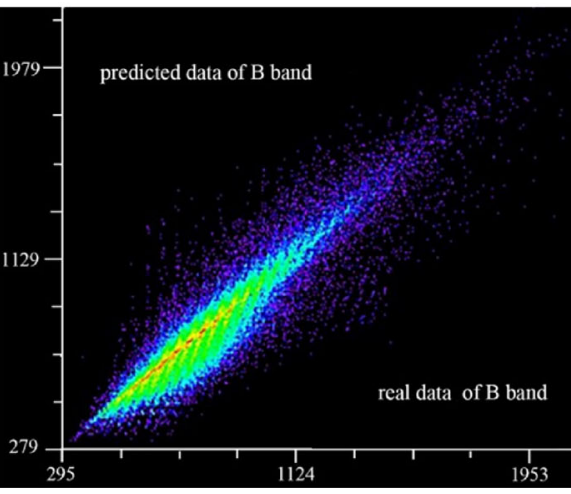

C

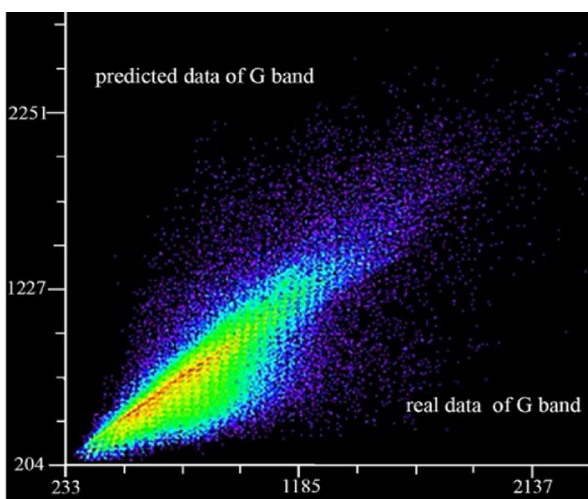

b

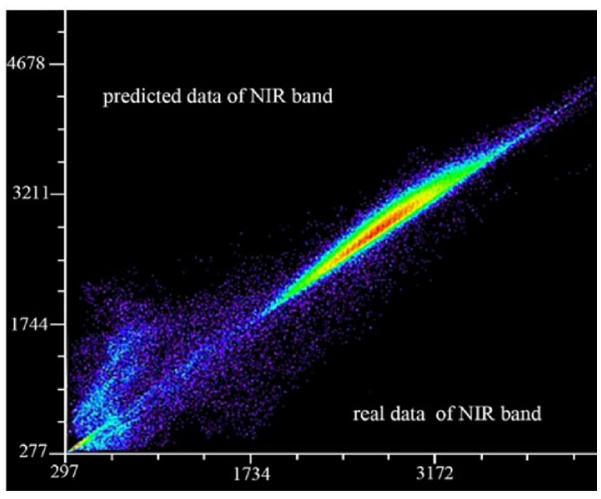

d

Fig. 2 The correlation analysis of fusion forecast reflectance data with TM. a Correlation between real data and predicted data of R band. $\mathbf{b}$ Correlation between real data and predicted data of $\mathrm{G}$ band. $\mathbf{c}$ Correlation between real data and predicted data of B band. $\mathbf{d}$ Correlation between real data and predicted data of NIR band

of NDVI and NDWI between the two time phases were analyzed through superposition. NDVI as a commonly used vegetation index can effectively reflect the vegetation information $[20,21]$. Due to the vast areas and diverse types of water bodies (big and small rivers, reservoirs, ponds, shallow swamps) in the wetlands of
Panjin, we introduced NDWI to reflect the distribution of water bodies.

Analysis of experimental results shows the NDVI of a part of pixels exceeds the range $(-1,1)$, which is because of errors of atmospheric correction in the FLAASH of TM images as well as the trimmed boundaries. The

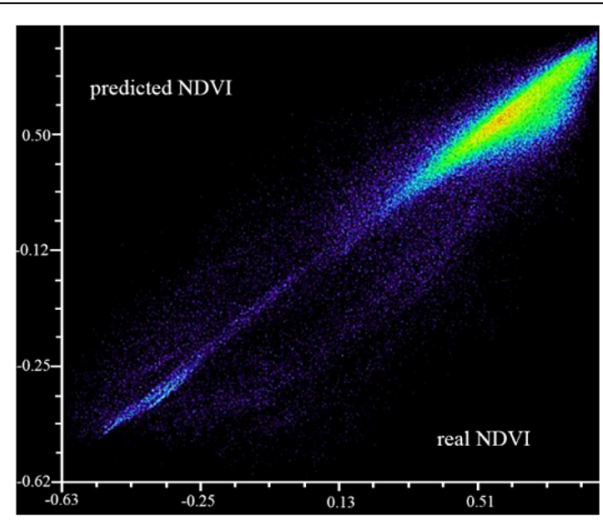

a

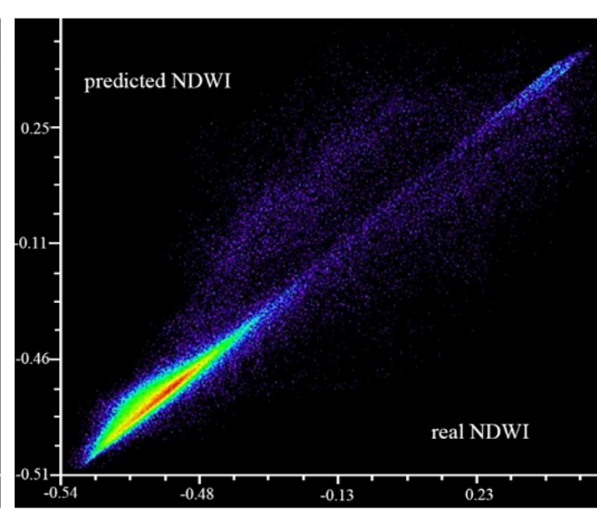

b

Fig. 3 The correlation analysis of fusion forecast data with NDVI and NDWI. a Correlation between real NDVI and predicted NDVI. b Correlation between real NDWI and predicted NDWI 


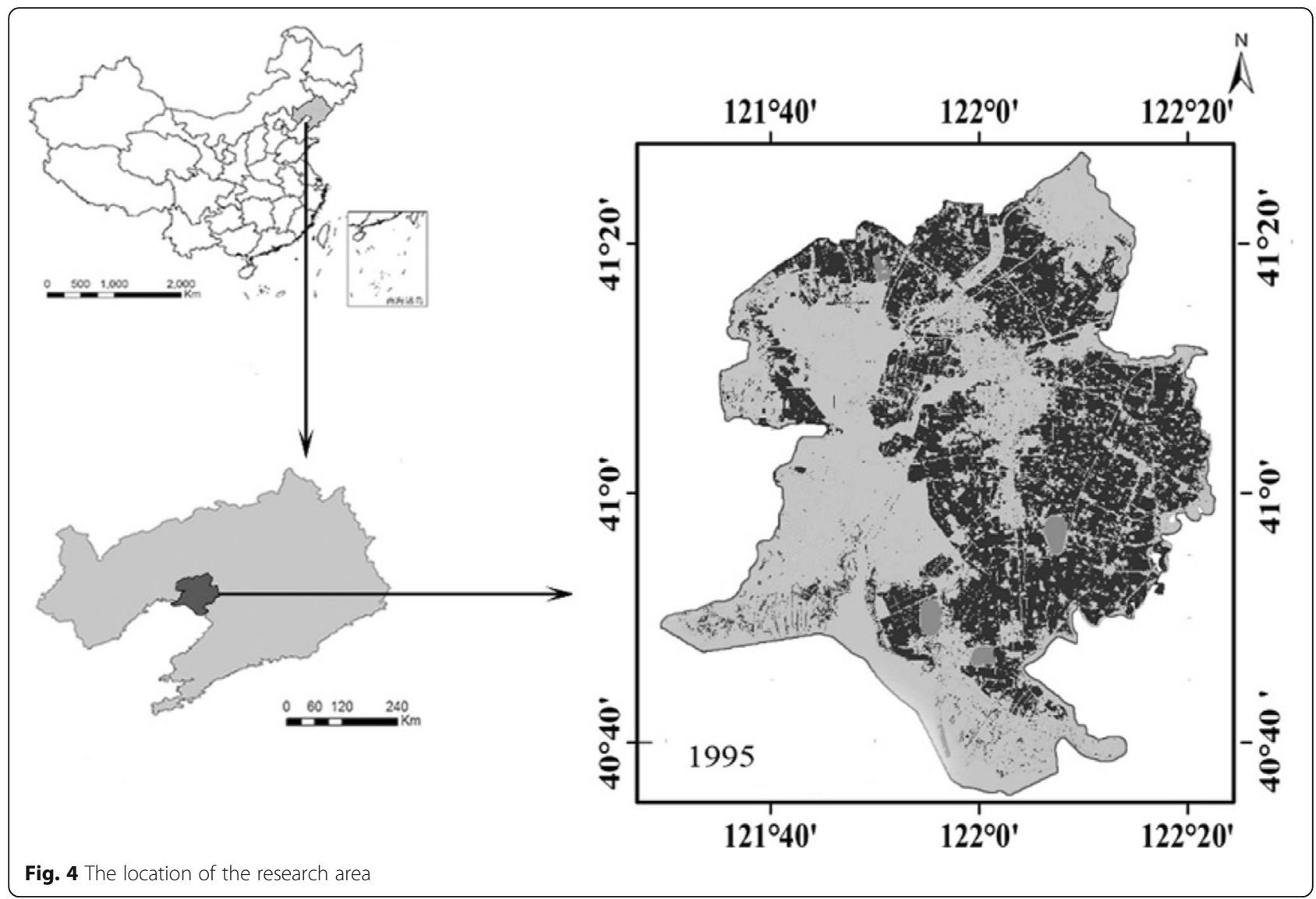

abnormal data were removed. Specifically, values $>1$ were assigned as " 1 ," and the values $<1$ were treated as "- 1." The abnormal data were calculated as $-1.0>$ b1 $<$ 1.0 by using the Band Math on ENVI (Fig. 6).

Clearly, in June and August, the majority of Panjin was covered by vegetation, and a part of the water bodies were even found with large NDVI, which indicate the large chlorophyll concentrations in the water bodies. Data superposition between two phases (data in $\mathrm{Au}-$ gust-data in June) can reveal the changes of NDVI and NDWI. Clearly, the NDVI throughout Panjin increases but NDWI decreases from June to August. Meteorological data show heavy rainfall $(321 \mathrm{~mm})$ occurred in June 2016 in Panjin, but the rainfall in August was smaller $(160 \mathrm{~mm})$. The experimental results are basically consistent with the real data.

\subsection{Changes of vegetation coverage}

Vegetation coverage measures the proportion of vertical projected areas (leaves, stems, branches) to the total area of the study site. Vegetation coverage well reflects the seasonal changes of vegetation growth, ecological environment, water/soil quality, and water conditions in wetlands and is a favorable indicator of seasonal changes in wetlands. The development of RS offers powerful technical support for estimation of large-area regional vegetation coverage. Many methods can be used to detect and calculate vegetation coverage with the help of RS. Here, an improved model based on pixel equinoctial model through experiments was used.

To extract more accurate vegetation coverages, we first used two phases of NDVI (set NDVI $>0.0$ ), firstly extracted the vegetation information with the preset threshold, and then computed vegetation coverage. The vegetation coverages on 18 June and 16 August 2016 in Panjin are showed below. Clearly, the majority of Panjin was covered by vegetation; the overall vegetation coverage in June was smaller than in August, which was mainly because vegetation grew further under sufficient rainfall, and rice, reed, and other crops grew well.

The vegetation coverage rates in Panjin and its districts and counties were summarized on ArcGIS. The average vegetation coverage rate in the whole urban areas of Panjin on 18 June 2016 was 0.71 and rose by 0.02 to 0.73 in 16 August. Statistics by districts and counties showed the vegetation coverage rates in June were all smaller than in August and were smaller in urban areas than in other areas.

To facilitate statistics and analysis, we further divided vegetation coverage into six levels: bare areas $(0,0.1)$, 


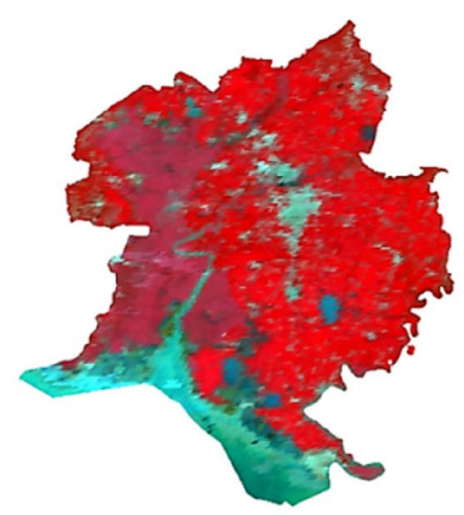

$\mathbf{a}$

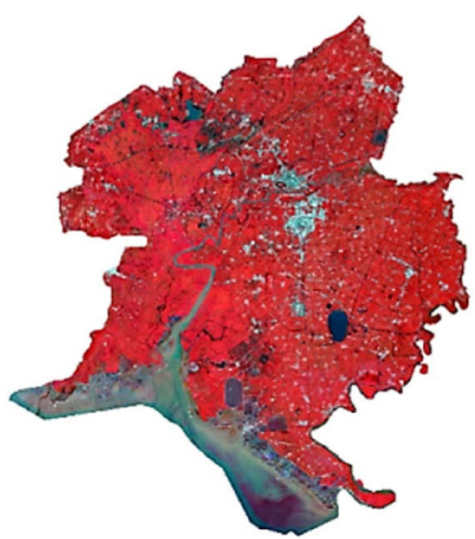

c

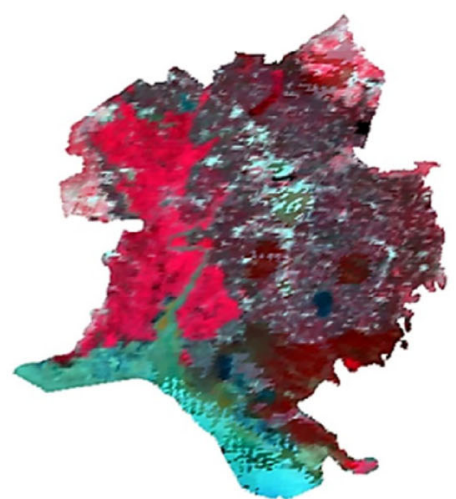

b

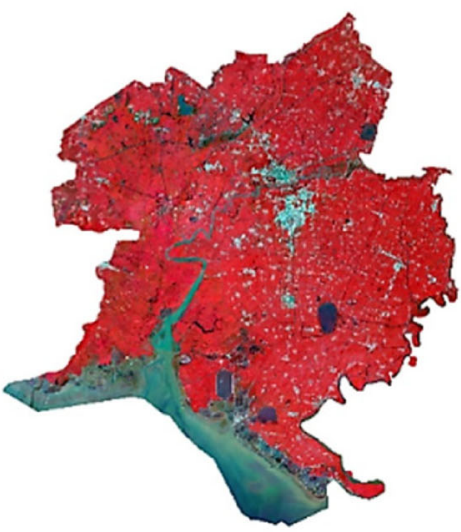

d

Fig. 5 The false color MODIS image of composite original and merged in Panjin. a Original MODIS images on 18 June 2016. b Original MODIS images on 16 August 2006. c Merged MODIS images on 18 June 2016. d Merged MODIS images on 16 August 2006

low coverage $[0.1,0.3)$, medium to low coverage $[0.3$, $0.45)$, medium coverage $[0.45,0.6)$, and high coverage $[0.6$, $1)$. Then, the divisions were segmented by colors and densities to classify the vegetation coverage rates (Fig. 7). Clearly, the whole city in 2016 was dominated by high to medium coverage. The vegetation coverage rate in the wetlands was higher in August than in June. The land covers with high coverage $(>0.6)$ were reeds and rice lands, and the land covers with low coverages included beaches and sea shores $(0.1-0.3)$. The vegetation coverage of wetland vegetation especially reeds rose rapidly.

Previous studies and this study suggest wetland resources are largely influenced by climate, and especially, the changeable meteorological conditions in Panjin between June and September with cloudy and rainy climate make the corresponding Landsat images unavailable. The RS space-time fusing technique provides an effective way to solve the seasonal changes of wetlands, to modestly overcome the limitations of traditional investigation methods, and solve the technical problems in applications of wetland resource investigation.

\section{Discussion}

The RS space-time fusing technology makes full use of the advantages of different information sources. The improved STARFM generates high-precision multi-temporal RS data, which provide a new valid RS source for land resource RS-based investigation. It is experimentally validated that MODIS and Landsat images can be well fused and the fused images are highly correlated with the TM images. In particular, the correlations rank by NDWI $(0.9678)>$ green band $(0.9630)>$ near-infrared band $(0.9345)>$ blue band (0.9018). This method can be referred to in traditional RS-based land resource investigations, including wetlands.

Due to influences by climate, terrains, and soils, the wetland resources are complex and changeable. Moreover, the majority of wetlands are geographically remote and traffically inconvenient, so the traditional monitoring methods are limited by the huge material costs, vast investigation extent, destruction to wetlands, long investigation period, and low time effectiveness. In 

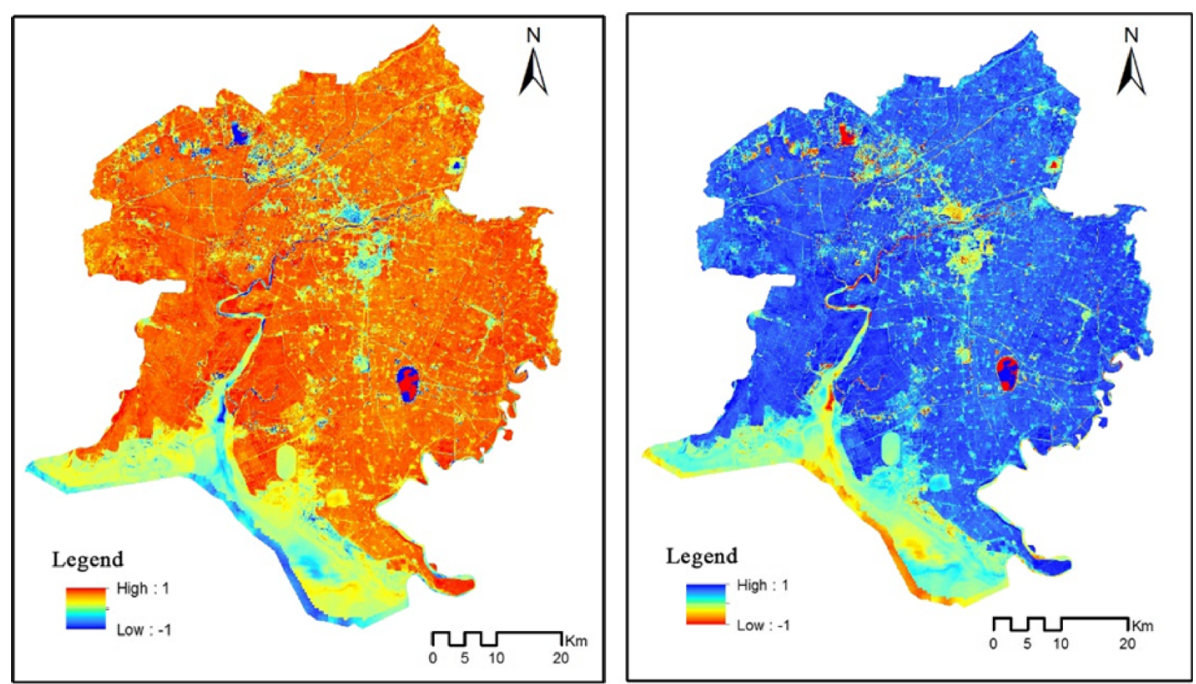

a The exponential distribution of NDVI and NDWI in June 18, 2016,
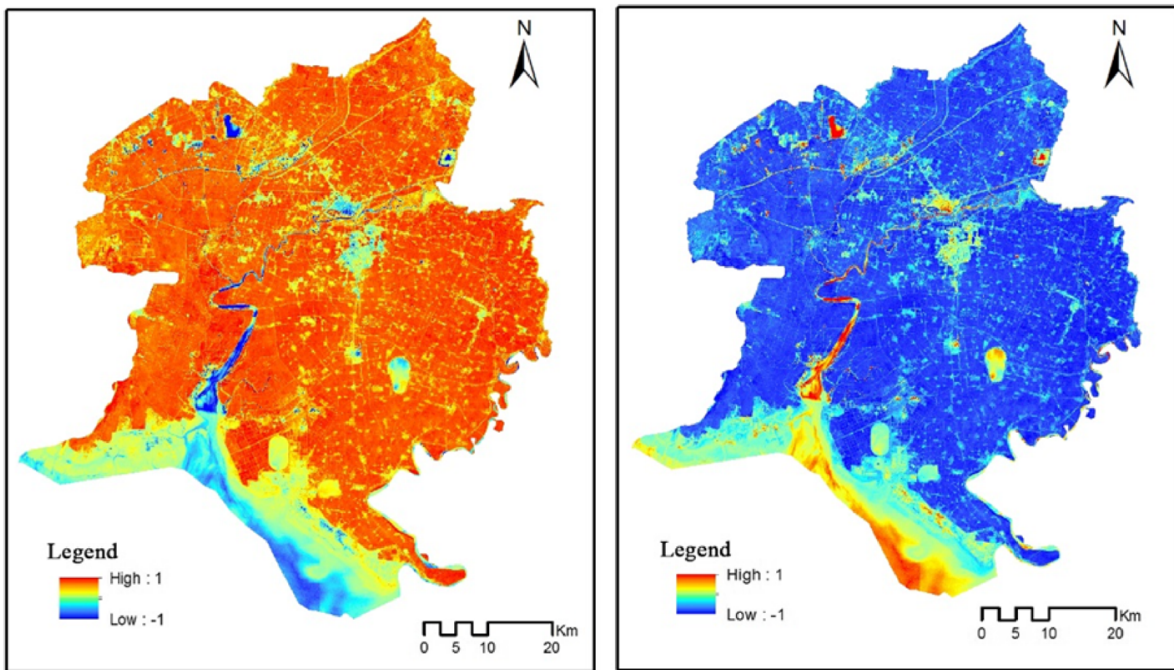

b The exponential distribution of NDVI and NDWI in August 16, 2016

Fig. 6 a The exponential distribution of NDVI and NDWI in 18 June 2016, b The exponential distribution of NDVI and NDWI in 16 August 2016

this study, data fusion was used to study the seasonal changes of wetlands in Panjin. The two phases of RS images in June and August 2016 were inverted. It was found that the fused images well reflected the seasonal changes of NDVI, NDWI, and vegetation coverage in the wetlands and modestly solved the limitations in the cloudy and rainy climate.

\section{Conclusions}

The RS space-time fusing technology can be used to study the dynamic changes in the distribution, types, and areas of wetlands, to investigate the distribution, trends, and spatial patterns of wetland biological resources and to invert the daily or seasonal changes of near-ground atmospheric $\mathrm{CO}_{2}$ concentrations, vegetation growing trend, hydrological characteristics, surface suspended matter concentrations, and spatial patterns in the wetlands.

In the high-water period (June to September) of wetlands, the presence of cloud and rain leads to massive data loss and useless Landsat images. The traditional RS methods for classifying and extracting regional wetlands would cause severe interferences. Thus, an effective way is to use STARFM to fuse MODIS and Landsat images, solving the problem of data loss, and thereby to extract and monitor the wetland-related information. However, when an RS space-time fusing method is used to extract information of wetlands, both the phenologic changes and space information of wetlands should be taken into account, especially in regions with severe space heterogeneity. Thus, one trend in the future is to converse 

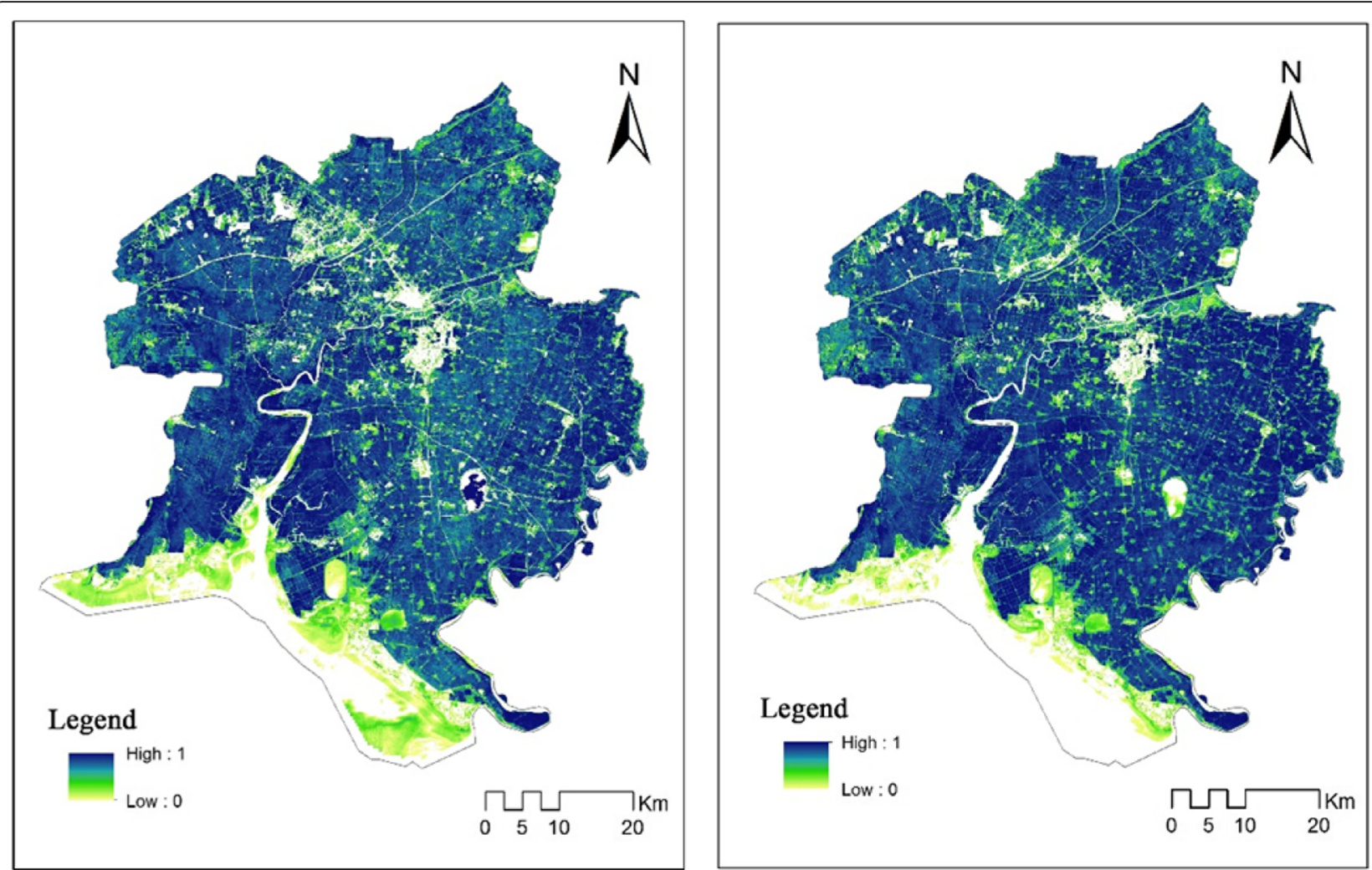

Fig. 7 The vegetation coverage map of Panjin between 18 June and 16 August 2016

scale or fuse other RS data and thereby to effectively solve the problem of space heterogeneity.

Influenced by factors such as climate, topography, and soil, wetland resources possess the characteristics of complex and changeable land types, and the geographical location of most wetlands is remote with poor transportation. Only relying on conventional means to monitor wetland situation not only requires a large amount of material cost with small investigation scope, but also causes damage to the wetland, which has a long period of wetland research and insufficient timeliness. The time-space fusion of remote sensing data effectively gives play to the advantages of different information sources of MODIS and Landsat data; the remote sensing information source obtained by modified STARFM model fusion algorithm has the advantages of multidate, high precision, etc., providing an effective remote sensing information source for remote sensing investigation of land resource. Experiment proves that MODIS and Landsat data can be fused in a good manner, the image after fusion has high correlation with TM image, and this method can provide practical reference for the remote sensing investigation of traditional land resources including wetland resource. Research results show that the image after fusion can well reflect the vegetation index, water body index, and vegetation coverage of the wetlands' seasonal variation, which solve the research problem of wetland seasonal characteristics in cloudy and rainy weather to some extent.

\section{Abbreviations}

CDSTRFMA: Combination of Downscaling Mixed Pixel Algorithm and Spatial and Temporal Adaptive Reflectance Fusion Model; MODIS: Moderateresolution imaging spectroradiometer; NASA: National Aeronautics and Space Administration; NDVI: Normalized Difference Vegetation Index; NDWI: Normalized Difference Water Index; RS: Remote sensing;

STARFM: Spatial and Temporal Adaptive Reflectance Fusion Model;

TM: Thematic Mapper

\section{Acknowledgements}

The corresponding author is professor Wang Qiubing. We thank the USGS and the Remote Sensing Data Sharing Center of China for providing Landsat data.

\section{Funding}

Completed research programs were supported by the National Natural Science Foundation of China (No. 41771245, No. 40671079, No. 40971124, and No. 41371223).

Availability of data and materials

We can provide the data.

\section{Authors' contributions}

FM and QW conceived of and designed the study and performed the experiments. FM and MZ analyzed and interpreted the data. QW and FM drafted the paper. All authors gave final approval of the version to be published. 
Ethics approval and consent to participate

Not applicable.

\section{Consent for publication}

Not applicable.

\section{Competing interests}

The authors declare that they have no competing interests.

\section{Publisher's Note}

Springer Nature remains neutral with regard to jurisdictional claims in published maps and institutional affiliations.

Received: 26 April 2018 Accepted: 10 July 2018

Published online: 27 July 2018

\section{References}

1. J William, GG James, Wetlands [M] (Wiley, New York, 2000)

2. A Ghermandi, JCJM van den Bergh, et al., Values of natural and human-made wetlands: a meta-analysis [J]. Water Resour. Res. 46(12), 137-139 (2010)

3. DM Kent, Applied wetlands science and technology [M] (Lewis Publishers Inc, Chelsea, 1996)

4. J Li, Z Bo, Z Leng, et al., Current status and prospect of researches on wetland monitoring based on remote sensing [J]. Prog. Geogr. 26(1), 33-43 (2007)

5. Z Guangming, Y Siyuan, L Guangxue, Application of satellite remote sensing to wetland research [J]. Mar. Geol. Lett. 23(12), 28-33 (2007)

6. Z Zhilong, Z Yili, L Linshan, et al., Advances in research on wetlands of the Tibetan Plateau [J]. Prog. Geogr. 09, 1218-1230 (2014)

7. K Jia, X Wei, X Gu, et al., Land cover classification using Landsat 8 Operational Land Imager data in Beijing, China [J]. Geocarto Int. 29(8), 941-951 (2014)

8. P Jönsson, L Eklundh, Timesat-a program for analyzing time-series of satellite sensor data [J]. Comput. Geosci. 30(8), 833-845 (2004)

9. H Hiker, MA Wulder, NC Coops, et al., Generation of dense time series synthetic Landsat data through dada blending with MODIS using a spatial and temporal adaptive reflectance fusion model [J]. Remote Sens. Environ. 113(9), 1988-1999 (2009)

10. F Gao, J Masek, M Schwaller, et al., On the blending of the Landsat and MODIS surface reflectance: predicting daily Landsat surface reflectance [J]. IEEE Trans. Geosci. Remote Sens. 44(8), 2207-2218 (2006)

11. Z Wei, A Li, J Hua-an, et al., An enhanced spatial and temporal data fusion model for fusing Landsat and MODIS surface reflectance to generate high temporal Landsat-like data []]. Remote Sens. 5(10), 5346-5368 (2013)

12. $\mathrm{M}$ Wu, N Zheng, C Wang, Assessing the accuracy of spatial and temporal image fusion model of complex area in South China [J]. J. Geo-Inf. Sci. 05, 776-783 (2014)

13. XX Han, XL Chen, F Lian, et al., Four decades of winter wetland changes in Poyang Lake based on Landsat observation between 1973 and 2013 [J]. Remote Sens. Environ. 156, 426-437 (2015)

14. X Dengfeng, Z Jinshui, S Peijun, et al., Remote sensing data fusion by combining STARFM and downscaling mixed pixel algorithm [J]. J. Remote Sens. 20(1), 62-72 (2016)

15. JJ Walker, KM Beurs, RH Wynne, et al., Evaluation of Landsat and MODIS data fusion products for analysis of dryland forest phenology [J]. Remote Sens. Environ. 117(1), 381-393 (2012)

16. J Kun, L Shunlin, Z Ning, et al., Land cover classification of finer resolution remote sensing data integrating temporal features from time series coarser resolution data. J. Photogramm. Remote Sens. 93(5), 49-55 (2014)

17. H Pengyu, W Li, N Zheng, et al., The potential of time series merged from Landsat-5 TM and HJ-1 CCD for crop classification: A case study for Bole and Manas counties in XinJiang, China []]. Remote Sens. 6(8), 7610-7631 (2014)

18. H Jiang, C Liu, X Sun, et al., Remote sensing reversion of water depths and water management for the stopover site of Siberian cranes at Momoge, China [J]. Wetlands 35(2), 369-379 (2015)

19. X Li, Z Xue, J Gao, Dynamic changes of plateau wetlands in Madou County, the Yellow River source zone of China: 1990-2013 []]. Wetlands 36(2), 299-310 (2016)

20. S Liang, Automatic land-cover update approach integrating iterative training sample selection and a Markov Random Field model [J]. Remote Sens. Lett. 5(2), 148-156 (2014)

21. CQ Huang, Y Peng, M Lang, et al., Wetland inundation mapping and change monitoring using Landsat and air-borne LiDAR data [J]. Remote Sens. Environ. 141, 231-242 (2014)

\section{Submit your manuscript to a SpringerOpen ${ }^{\circ}$ journal and benefit from:}

- Convenient online submission

- Rigorous peer review

- Open access: articles freely available online

- High visibility within the field

- Retaining the copyright to your article

Submit your next manuscript at $\boldsymbol{\nabla}$ springeropen.com 